\title{
The economic burden of influenza-like illness among children, chronic disease patients and the elderly in China: a national cross-sectional survey
}

\author{
Xiaozhen Lai ${ }^{1}$, Hongguo Rong ${ }^{1}$, Xiaochen $\mathrm{Ma}^{1}$, Hou Zhiyuan ${ }^{2}$, Shunping $\mathrm{Li}^{3}$, Rize Jing ${ }^{1}$, \\ Haijun Zhang ${ }^{1}$, Yun Lyu ${ }^{1}$, Jiahao Wang ${ }^{1}$, Huangyufei Feng ${ }^{1}$, Zhibin Peng ${ }^{4}$, Luzhao Feng ${ }^{5}$, \\ and Hai Fang ${ }^{1}$ \\ ${ }^{1}$ Peking University Health Science Centre \\ ${ }^{2}$ Fudan University \\ ${ }^{3}$ Shandong University \\ ${ }^{4}$ Chinese Center for Disease Control and Prevention \\ ${ }^{5}$ School of Population Medicine and Public Health, Chinese Academy of Medical Sciences \\ \& Peking Union Medical College
}

August 20, 2020

\begin{abstract}
Background: The disease burden of seasonal influenza is substantial in China, while there lacks nation-wide economic burden estimates. This study aims to examine influenza-like illness (ILI) prevalence, healthcare seeking behaviors, economic impact of ILI and its influencing factors among children, chronic disease patients and the elderly during the 2018-19 influenza season. Methods: From August to October 2019, 6668 children's caregivers, 1735 chronic disease patients, and 3849 elderly people were recruited from ten provinces in China to participate in an on-site survey. The economic burden of ILI consisted of direct (medical or non-medical) and indirect burden, and multivariate linear regression was adopted to predict the influencing factors of total economic burden. Results: There were $45.73 \%$ children, $16.77 \%$ chronic disease patients and $12.70 \%$ elderly people reporting ILI, and most participants chose "Outpatient service only", "Over-the-counter (OTC) medication only" or "Outpatient + OTC" after ILI. The average total economic burden of ILI was 1848 yuan (USD266.1) for children, 1105 yuan (USD159.1) for chronic disease patients and 2064 yuan (USD297.2) for the elderly. Multivariate linear regression showed that boys, the only child in family, and urban adult residents tended to have larger economic burden after ILI. Conclusions: Large economic burden of ILI was highlighted, especially among the elderly with less income and larger medical burden, as well as children with higher prevalence and higher self-payment ratio. It is important to adopt targeted interventions for high-risk groups, and this study can help national-level decision-making on the introduction of influenza vaccination as public health project.
\end{abstract}

\begin{abstract}
:
Background: The disease burden of seasonal influenza is substantial in China, while there lacks nation-wide economic burden estimates. This study aims to examine influenza-like illness (ILI) prevalence, healthcare seeking behaviors, economic impact of ILI and its influencing factors among children, chronic disease patients and the elderly during the 2018-19 influenza season.

Methods: From August to October 2019, 6668 children's caregivers, 1735 chronic disease patients, and 3849 elderly people were recruited from ten provinces in China to participate in an on-site survey. The economic burden of ILI consisted of direct (medical or non-medical) and indirect burden, and multivariate linear regression was adopted to predict the influencing factors of total economic burden.
\end{abstract}


Results: There were $45.73 \%$ children, $16.77 \%$ chronic disease patients and $12.70 \%$ elderly people reporting ILI, and most participants chose "Outpatient service only", "Over-the-counter (OTC) medication only" or "Outpatient + OTC" after ILI. The average total economic burden of ILI was 1848 yuan (USD266.1) for children, 1105 yuan (USD159.1) for chronic disease patients and 2064 yuan (USD297.2) for the elderly. Multivariate linear regression showed that boys, the only child in family, and urban adult residents tended to have larger economic burden after ILI.

Conclusions: Large economic burden of ILI was highlighted, especially among the elderly with less income and larger medical burden, as well as children with higher prevalence and higher self-payment ratio. It is important to adopt targeted interventions for high-risk groups, and this study can help national-level decision-making on the introduction of influenza vaccination as public health project.

Key words: Economic burden; Influenza-like illness; healthcare seeking behaviors; China.

\section{Background}

Annual influenza epidemics result in substantial morbidity and mortality across the globe, with a large share of the total disease burden occurring in low- and middle-income countries (LMICs). ${ }^{[1]}$ As estimated by the World Health Organization (WHO) in 2018, the annual epidemics of seasonal influenza could result in about 3 to 5 million severe cases and 290-650 thousand influenza-associated deaths. ${ }^{[2]}$ The disease burden of seasonal influenza is also substantial in China. A recent study estimated the influenza-associated excess respiratory mortality in mainland China, and found that about 88 thousand influenza-associated excess respiratory deaths occurred annually, corresponding to $8.2 \%$ of respiratory deaths. ${ }^{[3]}$

As suggested in the WHO position paper, those at particular risk of developing severe disease, i.e. disease resulting in hospitalization or death, or those at increased risk of exposure to influenza virus, were identified as risk groups for influenza, including children aged $<5$ years, the elderly, pregnant women, individuals with underlying health conditions, and healthcare workers. Illness severity and mortality are the greatest in highrisk groups, and so are the associated health care costs and productivity losses. Accordingly, the Technical Guidance for Influenza Vaccination in China (2019-20) clearly pointed out that children (aged between 6 to 59 months), patients with chronic diseases, and the elderly (aged 60 years old or above) are among the priority groups for influenza vaccination, together with healthcare workers, family members and caregivers of infants under 6 months, and pregnant women. ${ }^{[4]}$

However, influenza vaccination has not been included in China's National Immunization Program (NIP), and the expenses are paid totally out of pocket on most occasions, even for the priority groups. ${ }^{[5]}$ The coverage rate of influenza vaccination in China is extremely low in the past 15 years, with only $2 \%$ of the entire population being immunized, far lower than that of western developed countries and some developing countries in Asia and South America. ${ }^{[3]}$ In this case, it is important to consider the economic burden of influenza disease at the national level to make informed and evidence-based decisions.

Currently, sufficient data to precisely estimate the economic burden of influenza, especially in developing countries, are not available. A systematic review in 2015 suggested that information on the economic burden of influenza in LMICs was scarce and incomplete. ${ }^{[6]}$ In China, there were several studies examining the incidence and economic burden of influenza, but most of them were conducted at hospital or provincial level. ${ }^{[7-11]}$ Given the lack of economic burden estimates of seasonal influenza across China, the present study aims to examine influenza-like illness (ILI) prevalence, influenza-related healthcare seeking behaviors, the economic impact of ILI and its influencing factors among children, chronic disease patients and the elderly during the 2018-19 influenza season in ten Chinese provinces.

2. Methods

\subsection{Study population and sampling}

In August to October 2019, a total of 148 community health centers from 10 provinces in China were approached to join the national survey on the economic burden of season influenza among three priority 
groups, including children aged 6-59 months, chronic disease patients aged 18-59 years, and the elderly aged above 60 years (Financing Strategies of Influenza Vaccination in China, NCT04038333). ${ }^{[12]}$ For children aged 6-59 months, we asked their parents or grandparents who accompanied them to health centers to finish the compulsory immunization procedure. ${ }^{[4]}$ For chronic disease patients and the elderly, we asked them in health centers or gathered them in neighborhood committees. This study was ethically reviewed and approved by the Peking University Institutional Review Board (IRB00001052-19076), and written informed consent was obtained from individual or guardian participants.

We calculated the sample size under the assumption that the predicted prevalence of ILI would be $40 \%$ among children, $10 \%$ among the elderly, and $20 \%$ among those with chronic diseases. With an allowable error of $5 \%$, a sample size of 369,138 , and 246 was determined for the three groups in each province. To allow for disqualification of incomplete questionnaires, we increased the sample size by $10 \%$, with a final target sample population of 4059,1518 , and 2706 . In practice, we collected a larger sample size than expected to increase the reliability of the results.

The survey adopted a multistage sampling method. First, ten provinces/municipalities were selected based on the Division of Central and Local Financial Governance and Expenditure Responsibilities in the Healthcare Sector released by the State Council in 2018, which stratifies the 31 provinces/municipalities in mainland China into five layers. ${ }^{[13]}$ The division of expenditure responsibility between central and local governments differed across layers, with ratios of 8:2 (first layer), 6:4, 5:5, 3:7, and 1:9 (fifth layer). In terms of location, socioeconomic development, and accessibility, ten provinces/municipalities $(3,3,1,1$, and 2 in each layer) were chosen, with their location and 2018 per capita GDP rank (e.g., 1/31), as shown in Figure 1. Second, in each province/municipality, a capital city or well-developed district (in municipalities) and a non-capital city or less-developed district were selected. Third, two subdistricts/counties were chosen in each city or district, in which three or more immunization centers (in community health centers or township clinics) and the corresponding neighborhood committees were approached to participate in the survey.

\section{(Insert Figure 1 here)}

\subsection{Measures}

The on-site survey was conducted by trained interviewers using a specially-designed online PAD questionnaire system. Automatic logical proofreading was adopted in the online questionnaire to reduce input errors and missing values. In addition, interview recordings are uploaded and spot-checked by quality control personnel to find and correct problems in time. The structured online questionnaire can be divided into four parts: (1) socio-demographics such as age, gender, education level, household monthly per capita income, place of residence (urban or rural), self-reported health status, etc.; (2) whether had ILI in the past season; (3) health care seeking behaviors after ILI, including outpatient service, inpatient service, and over-the-counter (OTC) medication; (4) economic burden of the latest ILI in terms of direct (medical and non-medical) costs and indirect costs.

The economic burden of diseases consists of direct (medical or non-medical), indirect and intangible burdens. The direct medical burden refers to the costs medical services, and direct non-medical burden refers to the costs of supporting activities in the treatment process, including transportation, accommodation, nutrition, etc. The indirect economic burden is the loss of effective labor force caused by the disease. The intangible burden refers to the pain, anxiety, inconvenience, and subsequent decline in the quality of life to patients and their families. In view of the difficulty to measure intangible burden, studies on economic burden of diseases usually focus on direct and indirect burdens. ${ }^{[14]}$

According to the WHO standard ILI case definition, body temperature [?] $38{ }^{\circ} \mathrm{C}$ with either cough or sore throat was used in the present study to distinguish influenza from other respiratory illnesses. ${ }^{[15]}$ In the present study, for each interviewee (or their child aged 6-59 months) who reported ILI in the past season, their health care seeking behaviors and economic costs after the ILI were further examined. Direct medical costs were asked in terms of services types (outpatient services, hospitalization services, and/or OTC medication) and co-payment in each service type (self-paid expenses and medical insurance reimbursement). Direct non- 
medical costs were inquired from aspects of transportation, nutrition/food, accommodation, and nursing worker hiring. Indirect burden was obtained by asking the lost labor days of the respondents and their families, and calculating the economic cost based on the per capita GDP of each province in 2018. ${ }^{[16]}$

\subsection{Statistical analysis}

We produced summary statistics using frequencies and proportions for categorical variables, and means and standard deviations for continuous variables. The chi-square and Mann-Whitney tests were used to assess differences in sample characteristics. The economic burden values of ILI was displayed as means and 95\% confidence intervals (CI). Multivariate linear regression analysis was adopted to predict the influencing factors (including age, gender, household income, place of residence, etc.) of total economic burden (in log form), and results were shown as coefficients and $95 \%$ CIs. A two-sided p-value below 0.05 was considered statistically significant. The reported direct medical and non-medical costs were adjusted for provincial health care price index and consumer price index, respectively, according to the China Statistical Yearbook 2019. ${ }^{[16]}$ All data were analyzed using Stata version 14.0 (Stata Corp., College Station, TX, USA).

\section{Results}

\subsection{Study Sample Characteristics}

A total of 12252 valid questionnaires (6668 for children, 1735 for patients with chronic diseases, and 3849 for the elderly) were received, with an effective response rate of $99.80 \%$. Table 1 shows the general characteristics of participants in the analysis of influenza-like illness economic burden. Overall speaking, $45.73 \%$ of children, $16.77 \%$ of chronic disease patients and $12.70 \%$ of elderly people reported to have ILI in the past season. Among the children, there were slightly more boys than girls (3497 vs. 3171), and the caregivers of 5812 out of 6668 children reported good health status for their children. In the other two groups, patients with chronic diseases 50-59 years of age accounted for $77.8 \%$, and the elderly 60-79 years of age accounted for 91.9\%. More women participated in the survey in both groups, and the majority of them had fair or poor self-reported health status. We further compared the distribution of age and gender in the three groups with that in China Population and Employment Statistics Yearbook 2019 ${ }^{[17]}$ and found similar results, indicating the national representativeness of the population collected in this study.

Among children aged 6-59 months, older children had higher possibility of catching ILI than younger children $(p<0.01)$, and the only child in the family was less likely to have ILI than those living in families with more than one child $(p<0.01)$. Among chronic disease patients aged 18-59 years, those with younger age were more likely to catch ILI than older adults $(p<0.01)$. Among the elderly aged above 60 years, respondents with higher household monthly per capita income $(p<0.05)$, living in urban areas $(p<0.01)$, or having urban employee medical insurance $(p<0.01)$ had lower possibility of having ILI. In the three groups, respondents with fair or poor self-reported health status had higher risk of catching ILI than those with good self-reported health status $(p<0.01)$.

\section{(Insert Table 1 here)}

\subsection{Health care seeking behaviors}

Table 2 shows the distribution of health care seeking behaviors among 3829 participants after the latest ILI by calculating the number of ILI cases leading to outpatient visits, hospitalization, and OTC medication. As the table indicates, most participants chose "Outpatient service only", "OTC medication only" or "Outpatient + OTC" after ILI, accounting for $84.29 \%$ children, $84.53 \%$ chronic disease patients and $73.42 \%$ elderly people.

More specifically, when ILI occurred in children, $38.11 \%$ of them only seek outpatient service, $35.82 \%$ accepted a combination of outpatient treatment and OTC medication, and $10.36 \%$ only purchased OTC drugs outside the hospital. There were also 102 children (3.35\%) not receiving any medical services after ILI. In terms of service type, $83.96 \%$ of children received outpatient service after ILI, $12.36 \%$ had inpatient service, and 
$51.39 \%$ received OTC medication. As for chronic disease patients, $34.36 \%$ of them only seek outpatient service after ILI, $26.46 \%$ received outpatient treatment and OTC medication, and $23.71 \%$ only purchased OTC drugs. There were 20 chronic disease patients $(6.87 \%)$ not receiving any medical services after ILI. In terms of service type, $67.70 \%, 8.59 \%$ and $53.95 \%$ of chronic disease patients received outpatient service, inpatient service and OTC medication, respectively. For the elderly after ILI, $32.52 \%$ of them only seek outpatient service, $25.15 \%$ only received OTC medication, and $15.75 \%$ accepted a combination of outpatient treatment and OTC medication. There were 32 elderly people $(6.54 \%)$ not receiving any medical services after ILI. In terms of service type, $60.12 \%, 20.04 \%$ and $47.44 \%$ of elderly people received outpatient service, inpatient service and OTC medication, respectively.

\section{(Insert Table 2 here)}

\subsection{Economic burden of influenza-like illness}

Table 3 shows the economic burden of children, chronic disease patients, and the elderly for the latest ILI during the 2018-19 influenza season, in which direct medical and non-medical costs were adjusted for provincial health care price index and consumer price index, respectively, and indirect cost was calculated based on the per capita GDP of each province. As the results indicate, the economic burden of ILI for a child was about 1848 yuan (1 yuan = USD0.144 on 13th August 2020), including reimbursed medical expenses of 272 yuan, self-paid medical expenses of 997 yuan, direct non-medical expenses of 212 yuan, and indirect cost of 366 yuan. The average economic burden for chronic disease patients was about 1105 yuan, including reimbursed medical expenses of 335 yuan, self-paid medical expenses of 386 yuan, direct non-medical expenses of 102 yuan, and indirect cost of 283 yuan. The elderly had much higher average economic burden of about 2064 yuan, including reimbursed medical expenses of 766 yuan, self-paid medical expenses of 778 yuan, direct non-medical expenses of 162 yuan, and indirect cost of 358 yuan. For children and chronic disease patients, outpatient and inpatient costs accounted for the vast majority of direct medical expenses, and nutrition/food and transportation accounted for most direct non-medical expenses. For the elderly, only inpatient cost accounted for the vast majority of direct medical expenses, and nutrition/food and accommodation accounted for most direct non-medical expenses.

\section{(Insert Table 3 here)}

\subsection{Influencing factors of total economic burden}

Table 4 shows the results of multivariate linear regression to predict the influencing factors of total economic burden (in log form), including age, gender, whether the only child in the family (only for children), household income, place of residence, basic medical insurance type, and self-reported health status. As 91 children, 16 chronic disease patients and 32 elderly people reported zero total economic burden, we calculated the $\log$ form for these respondents as $\log ($ total cost +1$)$.

Among children, the economic burden of boys (Coef. $=0.16,95 \%$ CI $0.04-0.29, \mathrm{p}<0.05)$ and the only child in the family (Coef. $=0.28,95 \%$ CI $0.15-0.41, \mathrm{p}<0.05)$ after ILI was larger than that of girls or those living in families with two or more children. In terms of self-reported health status, children reported to have good health status had smaller economic burden (Coef. $=-0.53,95 \%$ CI $-0.70-0.35, \mathrm{p}<0.05$ ) than those with fair or poor health status. Among chronic disease patients, those aged 50-59 years old suffered from lower economic burden after ILI (Coef. $=-1.09,95 \%$ CI $-2.01-0.17, \mathrm{p}<0.05$ ) compared with younger respondents who developed chronic diseases at earlier age. Besides, patients living in urban areas had smaller economic burden (Coef. $=-0.62,95 \%$ CI $-1.17-0.08, \mathrm{p}<0.05)$ than those living in rural areas. Among the elderly, the economic burden of urban residents (Coef. $=-0.77,95 \%$ CI $-1.29-0.25, \mathrm{p}<0.05$ ) and those reporting good health status (Coef. $=-0.68,95 \%$ CI $-1.16-0.21, \mathrm{p}<0.05$ ) after ILI was smaller than that of rural residents or those reporting fair or poor health status.

(Insert Table 4 here) 


\section{Discussion}

To the best of our knowledge, this is the first study using a nationally representative sample from ten provinces in China to investigate the economic burden of ILI among three high-risk groups including children, patients with chronic diseases, and the elderly. This study estimated the prevalence of self-reported ILI, ILI-related health care seeking behaviors, the economic burden of ILI and its influencing factors among children, chronic disease patients and the elderly in China.

Despite vaccination recommendations targeted to high-risk groups and their contacts, high prevalence of ILI (45.73\% of children, $16.77 \%$ of chronic disease patients and $12.70 \%$ of elderly people) was found among high-risk groups, especially among children aged 6-59 months. The results were similar to those reported in a previous study. ${ }^{[8]}$ As for the health care seeking behaviors after occurring ILI, "Outpatient service only", "OTC medication only" or "Outpatient + OTC" was most frequently chosen after ILI. At the same time, the health care seeking behaviors showed differences across the three high-risk groups. Firstly, the elderly $(20.04 \%)$ were more likely to receive inpatient service than children $(12.36 \%)$ and chronic disease patients $(8.59 \%)$, indicating that older ILI patients were at higher risk of becoming severe cases. Secondly, larger portion of chronic disease patients $(6.87 \%)$ and the elderly $(6.54 \%)$ did not receive any medical services after ILI compared with children (3.35\%), reflecting the problem that high-risk groups, especially the elderly and chronic disease patients, did not attach much importance to their health conditions after ILI. Consistent with a previous study in which $86 \%$ patients aged 60 years and above received ambulatory care only, and $14 \%$ were hospitalized. ${ }^{[11]}$

After the occurrence of ILI, the economic burden of the elderly was the highest (2064 yuan), followed by children (1848 yuan) and chronic disease patients (1105 yuan). It has been indicated that outpatient service and OTC medication were most frequently chosen after ILI for the three groups, but the results of medical expenses demonstrated that once inpatient service was used due to severe cases or other complications, the medical burden of ILI would be greatly increased in terms of both reimbursed expenses and self-paid expenses. A study published in 2007 estimated the medical and indirect costs attributable to annual influenza epidemics in the United States using publicly available epidemiological data, and found that indirect costs including lost productivity from missed work days and lost lives comprised even larger amount of economic burden of influenza than hospitalization costs. ${ }^{[18]}$ In the present study, direct medical and non-medical costs comprised larger amount of economic burden of ILI, perhaps due to the fact that we only included lost productivity from missed work days into indirect cost.

There were several studies examining the incidence and economic burden of influenza at hospital or provincial level in China. A population-based household survey conducted in 2007 in Guangdong Province found annual ILI incidence of $49.87 \%$ among children aged $1-4$ years and $2.99 \%$ among the elderly aged $>60$ years, and the mean medical cost of one episode was 172.5 yuan for residents of all age groups. ${ }^{[8]}$ A prospective study conducted in 2011-12 season among children younger than 5 years in Suzhou reported that the mean direct and indirect costs per episode of influenza were 777.4 yuan for outpatient clinics and 848.0 yuan for emergency departments. ${ }^{[9]}$ These results were similar to the ILI incidence and economic costs reported in the present study for the three vulnerable groups. As a rough estimate, the overall annual economic burden of ILI was about 69.4 billion yuan for children, 29.0 billion yuan for chronic disease patients and 43.7 billion yuan for the elderly based on the population size recorded in statistical yearbooks. ${ }^{[16-17,19]}$

As for the co-payment of medical costs, the self-payment ratio and self-paid expenses were higher among children than chronic disease patients and the elderly due to the design of insurance schemes. Moreover, compared with children and chronic disease patients, the elderly had a lower probability of catching ILI, but higher economic burden after catching ILI. A previous study also found that young children and the elderly accounted for over $70 \%$ of the economic burden of influenza-associated hospitalizations in Jingzhou, China, ${ }^{[20]}$ indicating the importance of targeted interventions such as vaccination against influenza and early treatment of ILI for high-risk groups, especially elderly people with less income and larger economic burden after ILI, as well as children with higher prevalence of ILI and higher self-payment ratio for medical expenses. 
Multivariate linear regression analysis indicated that boys and the only child in the family had larger economic burden after ILI, perhaps due to the son preference and child-centered culture in some nuclear families. ${ }^{[21-22]}$ As for adult respondents, it was found that urban residents had smaller economic burden than rural residents after ILI, which was consistent with some previous findings, ${ }^{[20]}$ while some other studies indicated that the medical costs of influenza among urban patients were higher than those for rural patients. ${ }^{\left[{ }^{[, 9]}\right.}$ Additionally, it was no wonder that for children and the elderly, the economic burden of those reporting good health status after ILI was smaller than that of those reporting fair or poor health status.

Immunization has been proven as one of the most cost-effective health investments to prevent and control influenza. ${ }^{[23]}$ However, the coverage rate of influenza vaccination in China is extremely low in the past 15 years, with only $2 \%$ of the entire population being immunized. ${ }^{[3]}$ Vaccination is widely recognized as an action with strong positive externalities, ${ }^{[24-25]}$ so public intervention is expected to drive vaccine coverage to a socially optimal level. ${ }^{[26]}$ However, as introduced above, currently, influenza vaccination has not been included in China's NIP, and the expenses are paid totally out of pocket on most occasions. Results derived from the present study on the economic burden evaluation of influenza can help raise awareness among the public health and clinical communities for the burden and consequences of this important disease. Moreover, it is crucial to support national-level decision-making on the introduction of influenza vaccination as public health project, complementary vaccination strategies and/or expanding vaccination target groups. ${ }^{[27]}$

The present study also has a few limitations. First, the economic burden reported in this study was collected from ILI cases instead of confirmed influenza cases. As recommended by WHO manuals, ILI sentinel surveillance data can be used to estimate the disease burden of influenza for specific risk groups, ${ }^{[28]}$ and sentinel surveillance on ILI cases is less specific but sensitive and rapid than laboratory surveillance. ${ }^{[29]}$ Therefore, it is a widely acceptable to use the results of ILI cases given that national-wide laboratory surveillance is not available at present in China, and population-based study can offer higher population representativeness than hospital surveillance data. Second, part of the elderly surveyed in this study were recruited from community health centers when they were seeking primary medical services, which may result in selection bias. Nevertheless, given the high prevalence of non-communicable chronic diseases among Chinese elderly ${ }^{[30]}$ and the fact that community health centers mainly provide primary health care, the bias can be reduced. Third, the self-reported responses may be subjected to recalling bias and a tendency to report socially desirable responses, therefore, the results should be interpreted with caution. To minimize its effect, we asked participants' most recent influenza episode. Despite these limitations, the nationally representative sample was large, with a diverse socio-demographic population, thus offering good generalizability for the three high-risk groups in China.

\section{Conclusions}

In conclusion, the prevalence of ILI was fairly high among children, chronic disease patients and the elderly during the 2018-19 influenza season in China, and most participants chose outpatient service and/or OTC medication after ILI. The results highlight the large economic burden of ILI, especially among the elderly with less income and larger economic burden after ILI, as well as children with higher prevalence of ILI and higher self-payment ratio for medical expenses. Besides, boys, the only child in the family, and urban adult residents tended to have larger economic burden after ILI. Therefore, it is important to adopt targeted interventions such as vaccination and early treatment for high-risk groups, and this study can help nationallevel decision-making on the introduction of influenza vaccination as public health project for the three high-risk groups.

\section{Declarations}

\section{Ethics approval and consent to participate}

This study was approved by Peking University Institutional Review Board (IRB00001052-19076). Written informed consent was obtained from individual or guardian participants.

\section{Availability of data and materials}


The datasets generated and/or analyzed during the current study are not publicly available because our informed consent form stated that the collected data would only be used for scientific research, but are available from the corresponding author on reasonable request.

\section{Competing interests}

The authors declare that they have no competing interests.

\section{Funding}

This study was supported in part by grants from the National Natural Science Foundation of China (71774006, 71981320749).

\section{Authors' contributions}

H.F., L.F., X.L., H.R., X.M., Z.H., S.L. and Z.P. designed the analysis. X.L., H.R., X.M., Z.H., S.L., R.J., H.Z., Y.L., J.W. and H.F. conducted the survey and collected the data. X.L. and H.F. did the data analysis. X.L. was a major contributor in writing the manuscript. H.F., L.F., X.M., Z.H., S.L. and X.L. reviewed and edited the paper. All authors contributed to the interpretation of the results, and to the revision and final preparation of the paper for submission.

\section{Acknowledgements}

We would like to thank Beijing, Shanghai, Jilin, Yunnan, Shandong, Guangdong, Jiangxi, Gansu, Chongqing and Henan Provincial/Municipal Centers for Disease Control and Prevention, all participating community health centers and community neighborhood committees for their contribution to this study.

\section{References}

[1] Newall AT, Chaiyakunapruk N, Lambach P, et al. WHO guide on the economic evaluation of influenza vaccination. Influenza Other Respir Viruses . 2018;12(2):211-219.

[2] World Health Organization. Influenza (seasonal) fact sheet November 2018. http://www.who.int/mediacentre/factsheets/fs211/en/. Accessed on 20 April 2020.

[3] Li L, Liu Y, Wu P, et al. Influenza-associated excess respiratory mortality in China, 2010-15: a populationbased study.Lancet Public Health . 2019;4(9):e473-e481.

[4] Technical guidelines for seasonal influenza vaccination in China (2019-20). Chin J Prev Med . 2020;54:2136. (in Chinese)

[5] Yang J, Atkins KE, Feng L, et al. Seasonal influenza vaccination in china: landscape of diverse regional reimbursement policy, and budget impact analysis. Vaccine . 2016;34(47):5724-5735.

[6] de Francisco Shapovalova N, Donadel M, Jit M, et al. A systematic review of the social and economic burden of influenza in low- and middle-income countries. Vaccine . 2015;33(48):6537-6544.

[7] Guo R, Zheng H, Huang L, et al. Epidemiologic and economic burden of influenza in the outpatient setting: a prospective study in a subtropical area of China. PLoS One. 2012;7(7):e41403.

[8] Guo R, Zheng H, Li J, et al. A population-based study on incidence and economic burden of influenza-like illness in south China, 2007. Public Health. 2011;125(6):389-395.

[9] Wang D, Zhang T, Wu J, et al. Socio-economic burden of influenza among children younger than 5 years in the outpatient setting in Suzhou, China. PLoS One. 2013;8(8):e69035.

[10] Zhang X, Zhang J, Chen L, et al. Pneumonia and influenza hospitalizations among children under 5 years of age in Suzhou, China, 2005-2011. Influenza Other Respir Viruses. 2017;11(1):15-22. 
[11] Chen J, Li Y, Gu B, et al. Estimation of the direct cost of treating people aged more than 60 years infected by influenza virus in Shanghai. Asia Pac J Public Health. 2015;27(2):NP936-946.

[12] Lai X, Rong H, Ma X, et al. Willingness to Pay for Seasonal Influenza Vaccination among Children, Chronic Disease Patients, and the Elderly in China: A National Cross-Sectional Survey. Vaccines.2020;8:405.

[13] The General Office of the State Council. Notice on the reform plan for the division of central and local financial governance and expenditure responsibilities in the healthcare sector. http://www.gov.cn/zhengce/content/2018-08/13/content_5313489.htm. Accessed on 11 January 2019.

[14] Drummond MF, O'Brien B, Stoddart GL, et al. Methods for the economic evaluation of health care programmes, 3rd ed. Oxford: Oxford University Press.

[15] Fitzner J, Qasmieh S, Mounts AW, et al. Revision of clinical case definitions: Influenza like illness and severe acute respiratory infection. Bull World Health Organ . 2018;96:122-128.

[16] National Bureau of statistics. China statistical yearbook 2019. China Statistics Press: Beijing, China, 2019 .

[17] Department of Population and Employment Statistics, National Bureau of Statistics of China. China Population \& Employment Statistics Yearbook 2019. China Statistics Press: Beijing, China, 2019.

[18] Molinari NA, Ortega-Sanchez IR, Messonnier ML, et al. The annual impact of seasonal influenza in the US: measuring disease burden and costs. Vaccine . 2007;25(27):5086-5096.

[19] National Health Commission of the People's Republic of China. China Health Statistics Yearbook 2019. Beijing: Peking Union Medical College Press, 2019.

[20] Yang J, Jit M, Leung KS, et al. The economic burden of influenza-associated outpatient visits and hospitalizations in China: a retrospective survey. Infect Dis Poverty . 2015;4(1):44.

[21] Barcellos SH, Carvalho LS, Lleras-Muney A. Child Gender and Parental Investments In India: Are Boys And Girls Treated Differently?.Am Econ J Appl Econ . 2014;6(1):157-189.

[22] Martinson ML, Chang YL, Han WJ, Wen J. Child Overweight and Obesity in Shanghai, China: Contextualizing Chinese Socioeconomic and Gender Differences. Int J Behav Med . 2018;25(1):141-149.

[23] Osterholm MT, Kelley NS, Sommer A, et al. Efficacy and effectiveness of influenza vaccines: a systematic review and meta-analysis. Lancet Infect Dis. 2012;12(1):36-44.

[24] Folland S, Goodman AC, Stano M. Epidemiology and Economics: HIV/AIDS in Africa. In The Economics of Health and Health Care, 7th ed.; Pearson Education: New Jersey, USA, 2012; pp. 531-548.

[25] Wilder-Smith A, Longini I, Zuber PL, et al. The public health value of vaccines beyond efficacy: methods, measures and outcomes.BMC Medicine. 2017;15(1):138.

[26] Chen F, Toxvaerd F. The economics of vaccination. J Theor Biol. 2014;363:105-117.

[27] World Health Organization. WHO Manual for Estimating the Economic

Burden of Seasonal Influenza, 2016. http://www.who.

int/immunization/documents/financing/who_ivb_16.04/en/. Accessed on 27 January 2020.

[28] World Health Organization. A Manual for Estimating Disease Burden Associated with Seasonal Influenza, 2015. http://apps.who.int/iris/bitstream/10665/178801/1/9789241549301_eng.pdf. Accessed on 28 January 2020.

[29] WHO recommended surveillance standards. 2nd ed. Geneva: World Health Organization, 1999. http://www.who.int/csr/resources/publications/surveillance/whocdscsrisr992.pdf. Accessed on 16 March 2020 . 
[30] Han M. Shi X, Cai C, et al. Evolution of non-communicable disease prevention and control in China. Glob Health Promot . 2017;26:90-95.

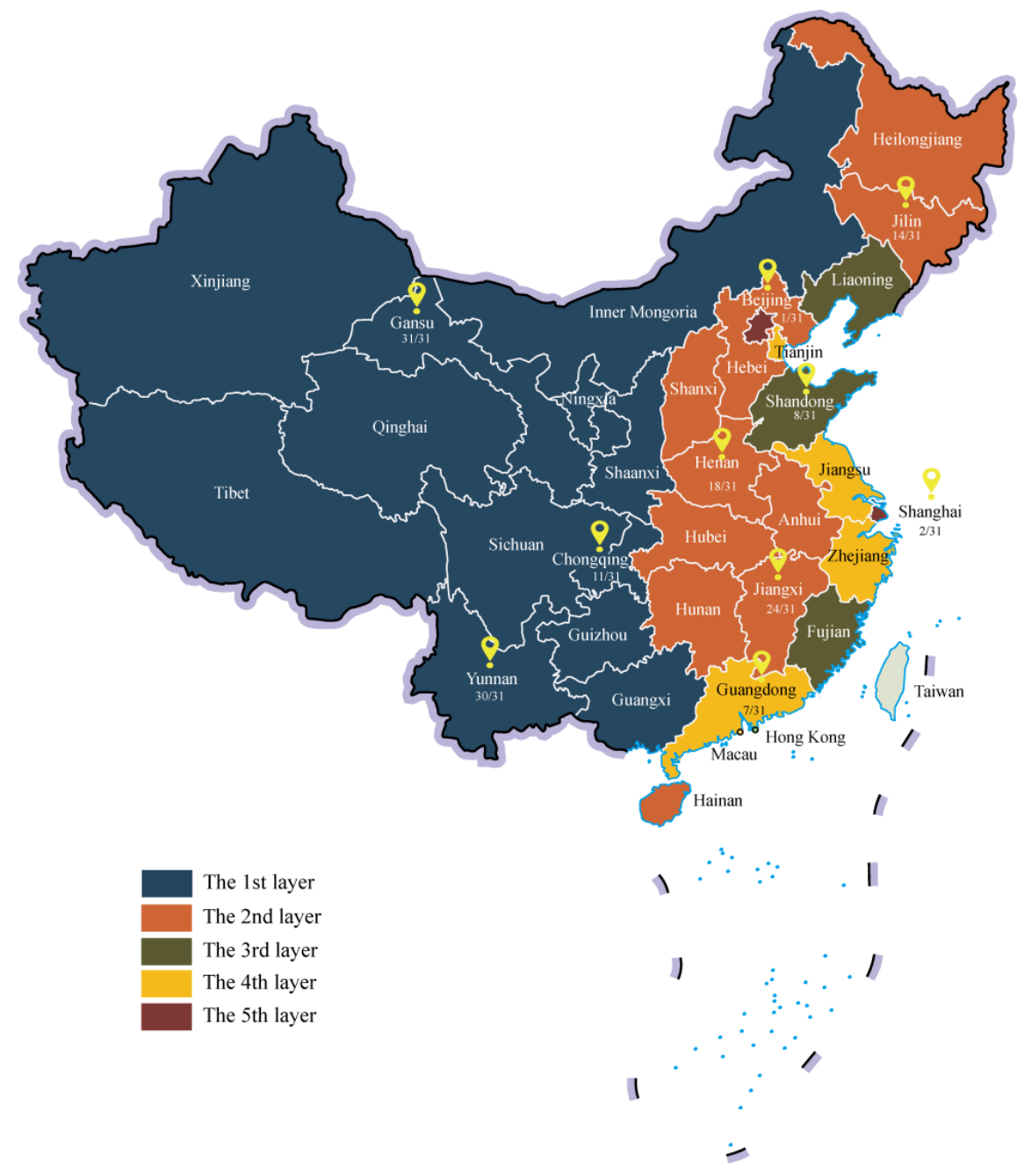

\section{Hosted file}

Table 1.docx available at https://authorea.com/users/351754/articles/476250-the-economicburden-of-influenza-like-illness-among-children-chronic-disease-patients-and-theelderly-in-china-a-national-cross-sectional-survey

\section{Hosted file}

Table 2.docx available at https://authorea.com/users/351754/articles/476250-the-economicburden-of-influenza-like-illness-among-children-chronic-disease-patients-and-theelderly-in-china-a-national-cross-sectional-survey

\section{Hosted file}

Table 3.docx available at https://authorea.com/users/351754/articles/476250-the-economicburden-of-influenza-like-illness-among-children-chronic-disease-patients-and-theelderly-in-china-a-national-cross-sectional-survey 


\section{Hosted file}

Table 4.docx available at https://authorea.com/users/351754/articles/476250-the-economicburden-of-influenza-like-illness-among-children-chronic-disease-patients-and-theelderly-in-china-a-national-cross-sectional-survey 